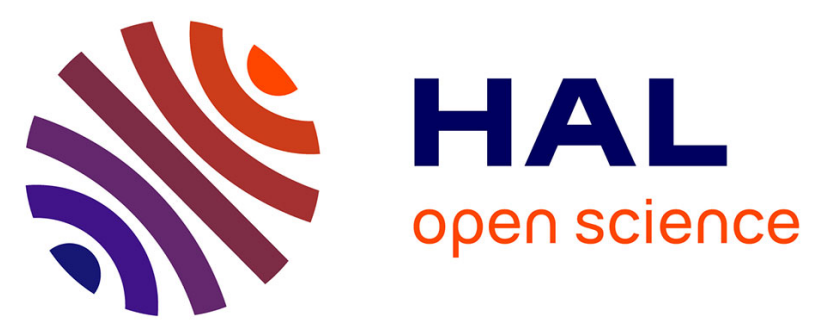

\title{
Prediction of transient chemistry effect during fuel pyrolysis on the pressure drop through porous material using artificial neural networks
}

Eddy El Tabach, Leyla Adishirinli, Nicolas Gascoin, Guillaume Fau

\section{- To cite this version:}

Eddy El Tabach, Leyla Adishirinli, Nicolas Gascoin, Guillaume Fau. Prediction of transient chemistry effect during fuel pyrolysis on the pressure drop through porous material using artificial neural networks. Third International Scientific Conference The Modeling Of Nonlinear Processes And Systems (MNPS-2015), Moscow State University of Technology "STANKIN" 22-26 June 2015, Moscow, Russia, 2015, moscou, France. 10.1016/j.jaap.2015.07.010 . hal-01253381

\section{HAL Id: hal-01253381 \\ https://hal.science/hal-01253381}

Submitted on 19 Feb 2016

HAL is a multi-disciplinary open access archive for the deposit and dissemination of scientific research documents, whether they are published or not. The documents may come from teaching and research institutions in France or abroad, or from public or private research centers.
L'archive ouverte pluridisciplinaire HAL, est destinée au dépôt et à la diffusion de documents scientifiques de niveau recherche, publiés ou non, émanant des établissements d'enseignement et de recherche français ou étrangers, des laboratoires publics ou privés. 


\section{Prediction of transient chemistry effect during fuel pyrolysis on}

2 the pressure drop through porous material using artificial neural

\section{networks}

$5 \quad{ }^{1}$ PRISME laboratory, University of Orleans, 63 avenue de Lattre de Tassigny, 18020 Bourges 6 Cedex, France.

$7 \quad{ }^{2}$ INSA Centre Val de Loire, PRISME laboratory, University of Orleans, 88 Boulevard 8 Lahitolle, 18000 Bourges, France.

9 * Corresponding author. Ph.D.; Tel.: +33 2 48238217; E-mail adress: eddy.el-tabach@univ10 orleans.fr (E. El Tabach)

\section{Abstract}

12 Hydrocarbon fuels appear as good candidates for cooling purpose within aerospace 13 applications. Fuel flows through permeable structures. Thus, internal convection cooling is 14 reinforced by chemical kinetics (endothermic effect of fuel pyrolysis). Perfectly tuned 15 conditions may thus rapidly change due to unexpected coke formation that will clogged the 16 pores of the material and thus strongly affect the cooling efficiency. The pressure drop is one 17 of the indicator to monitor the modification of the through-flow and thus of the cooling.

18 Having a tool to predict these variations is of practical and theoretical interest for a better

19 management of the complex chemical and physical phenomena. This paper presents a model 20 based on artificial neural networks (ANN) for estimating the transient changes of the pressure 21 drop of a reactive fluid (n-dodecane) under pyrolysis conditions passing through porous 
22 metal material. The ANN is developed using experimental data obtained from an 23 experimental bench, which assures the monitoring of fluid mass flow rate, pressure and 24 temperature in stationary and transient conditions. For each case, the fluid pressure which 25 crosses the metallic porous material is measured as a function of test time, inlet operating 26 pressure, temperature and fuel mass flow rate. The optimal ANN architecture with error 27 backpropagation (BPNN) was determined by the cross validation method. The ANN 28 architecture having 9 hidden neurons gives the best choice. Comparing the simulated values 29 by ANN with the experimental data indicates that the ANN model give correct results. The 30 performance of the ANN model is compared with the multiple linear regression model. This 31 work is expected to be used for later prediction of pressure drop under a wide range of 32 clogging conditions.

33 KEYWORDS: Pyrolysis; Artificial neural networks; Modelling; Permeation; Coke; Porous 34 medium. 


\section{Introduction}

The development of hypersonic vehicles for future access to space or civil transport applications leads to an important heating of the engine and air frame. At flight speeds near Mach 4 and above, the air taken on board these vehicles will be too hot to cool the engines and airframe. Therefore, using fuel within regenerative cooling technique may be applicable [1]. To do so, it will be necessary to study and develop adapted light weight and hightemperature materials whose characteristics in terms of permeability and porosity are well defined. Among the materials the composite ones made of Ceramic Matrix (e.g. silicon based matrix) with carbon fibers are particularly interesting. For the lowest speed regime, metallic materials may also be used [2]. The aero-thermal loads must be thus addressed to quantify permeability/porosity fluctuations of materials as a function of operating conditions.

In the literature, different studies are found in relationship with this need, experimentally [3-5] or numerically [6,7]; even mathematically [8]. Such studies are not only dedicated to the flow description but also to the heat transfers [9-11]. The flows in porous materials are widely studied under common operating conditions.

The problem becomes more difficult when the coolant can react with the materials or within the material (local coking) [2]. In case of chemical reaction, the formation of carbon deposit on the surface and inside the porosities can impact the physical properties of the material (lowering the permeability and the porosity) and thus the cooling efficiency. These reactions can be due to the thermal fluid decomposition and to the degradation of the material itself. The degree of decomposition is highly dependent on the operating conditions (temperature, pressure, type of flow, nature of reactor) [10,12-15]. Thermal cracking of hydrocarbons have been widely studied in petrochemical industry [16-20] and in the context of chemical vapor infiltration for the preparation of carbon/carbon composites [21-25]. It 
60

appears than the bigger the molecule, the higher the number of reactions which occur.

61 Considering dodecane pyrolysis, the number of reactions largely overpasses 1000 [11]. This implies very complex phenomena (heat and mass transfers with chemistry).

A lot of studies, often under high pressure (up to $2 \mathrm{MPa}$ ) are available for ambient to average temperature conditions (under $800 \mathrm{~K}$ ) [3] or for low pressure and high temperatures [1]. But only few are dedicated to both high temperature and high pressure conditions in case of reactive fluid. Numerous equations (derived from Brinkman's equation) which relate the pressure drop $\left(\triangle P=P_{\text {in }}-P_{\text {out }}\right)$ through the porous material to the through-flow velocity have been published $[4,6]$. They are based on coefficients, whose physical meaning is not evident [4]. One of the complexities of such configuration is due to the fact that along the chemical reactor (cooling channel of the hot vehicle), the fluid is supercritical [2]. Multi-species flow is found due to fuel degradation during which heavy compounds (coke particles) are formed and produce solid particles that can block the pore within the porous medium where they are flowing $[10,26]$. Due to these large and open difficulties, CFD calculations may not be relevant and experimental tests are costly and they cannot cover the entire range of test conditions/material variety, fluid nature.

As a consequence in this paper, we have used an approach based on the artificial neural networks (ANN) for simulating the transient changes of the pressure drop of n-dodecane (reactive fluid) passing through the porous material (Stainless Steel) by taking into account both high temperature and high pressure conditions. This work intends to indirectly predict the chemical effect of fuel pyrolysis, of coking and of clogging on the permeation process which directly controls the cooling efficiency. The description of the same numerical approach applied to another set of gas mixture (inert) and flow conditions can be found in a previous study [7]. Over the last two decades, ANN have been successfully used by many researchers for a wide range of engineering applications [27-29]. ANN is based on the 
substitution of the complex simulation model by an approximation of the input-output

86 relationship. ANN has the advantage over regression that the form of the model needs not to

87 be pre-determined [30]. In addition, ANN can theoretically approximate any function to any

88 level of accuracy, which is very interesting when the governing physical mechanisms are

89 non-linear like in high velocity fluid flow in porous materials. The database was built with

90 four input parameters (experiment time, inlet fuel mass flow rate, inlet operating pressure and 91 the uniform temperature) and with the outlet fuel pressure as the output parameter. The 92 results obtained experimentally are used to construct, to optimize and to validate the model.

93 This artificial neural network has been trained and tested on this database using the error 94 backpropagation algorithm and cross validation. The performance of the ANN model is 95 compared with a multilinear regression approximation method.

\section{2. Material}

$97 \quad 2.1$ Experimental permeation bench

98 The COMPARER pyrolysis test bench (Fig. (1)) is used to pressurize and to heat the fuel 99 under flow conditions [4]. Its main characteristics are the following:

100 - Maximum operating conditions: $1800 \mathrm{~K}, 8 \mathrm{MPa}, 0.0006 \mathrm{~kg} . \mathrm{s}^{-1}$ for liquid fuel and $0.006 \mathrm{~kg} \cdot \mathrm{s}^{-1}$ for gas.

- Sensors: 5 pressure transducers, 5 mass flow rates, over $10 \mathrm{~K}$-type and R-type thermocouples with data acquisition system (16 bits, 48 channels, $0.1 \mathrm{~Hz}$ ).

A permeation test cell contains the porous sample (Fig. 1). This cell is inserted inside the furnace of the COMPARER bench and it is connected to the fluid supply system and to the suitable sensors. The permeable material bounds the cell in two high and low pressure chambers (upstream and downstream to the porous material respectively). An inlet pipe provides the fuel into the system. This cell is connected to a dynamic sampling system to get 
109 hot pressurized samples at three location points in the cell. Despite its small size (external

diameter of $40 \mathrm{~mm}$ ), it enables measuring the temperature, pressure and mass flow rate on each side of the porous sample.

In the present work, an isotropic stainless steel material is preferred to composite one to avoid considering complex microstructure (fibres, layers). It is characterized by a porosity around $30 \%$, a grain diameter of $14.1 \mu \mathrm{m}$ and a pore diameter of $4.1 \mu \mathrm{m}$. Further geometrical information can be found in Gascoin et al. [4].

\section{Figure 1 should be placed here}

\subsection{Experimental test condition}

The different test conditions which were considered for the present work are the following:

- Temperature set-up: 3 different experimental test have been done for thermal plateau at $\mathrm{T}=725 \mathrm{~K}, 765 \mathrm{~K}$ and $810 \mathrm{~K}$. Each plateau last for about $30 \mathrm{~min}$ to one hour depending on the time requested by the system to reach steady-state conditions. Monitoring the entire test length enables getting transient evolution of all parameters.

- Absolute inlet pressure: in the range of [3.4 MPa ; 3.8 MPa].

- The experimental protocol is achieved with constant mass flow rate and given downstream pressure $\left(P_{\text {out }}\right)$. The upstream pressure $\left(P_{\text {in }}\right)$ increases due to coking and clogging of the porous medium; which makes the pressure drop to increase as a function of the test time.

- Monitoring of the chemical species: transiently thanks to a FTIR spectrometer for 5 gaseous species (methane, ethane, ethylene, propane, propylene) and during the three thermal plateaus by using a dedicated sampling system [10] coupled with a GC/TCD/FID/MS apparatus (more than 40 species analysed). 


\section{Experimental results}

We present in this section an experimental test result obtained for $\mathrm{T}=725 \mathrm{~K}$. As shown in Fig. 2, the measured pressure drop (Measured $P_{\text {in }}-P_{\text {out }}$ ) varies as a function of experimental time $(t)$ and the measured fuel mass flow rate $\left(q_{i n}\right)$ when the fuel (dodecane) temperature is kept constant $(\mathrm{T}=725 \mathrm{~K})$. Other obtained experimental results [10] showed that the temperature has a major effect on the measured pressure drop. Further details on the experimental results can be found in previous work [10]. Globally, based on the overall obtained experimental results, we can conclude that there are three parameters $\left(t, q_{i n}\right.$ and $\left.T\right)$ that have a great influence on the measurements of the pressure drop. These experimental results are necessary to construct, to optimize and to validate a model based on ANN for predicting the transient changes of the pressure drop of a reactive fluid (n-dodecane) passing through porous metal material (stainless steel). The construction of the developed ANN model is discussed in the following section.

\section{Figure 2 should be placed here}

\section{Construction of ANN models}

\subsection{Construction of the database}

ANN models learn the relationship between the input and the output parameters as a 50 result of training with previously recorded data. The database was built using experimental 151 data which are obtained from the developed experimental bench with input parameters: test 152 time $(t)$, operating inlet pressure $\left(P_{i n}\right)$, inlet fuel mass flow rate $\left(q_{\text {in }}\right)$ and temperature $(T)$ 153 varying in a range of representative values: between 0 and $858 \mathrm{~s}$ for $t$; between $3.3 \mathrm{MPa}$ and $1543.8 \mathrm{MPa}$ for $P_{i n}$; between $0.000033 \mathrm{~kg} / \mathrm{s}$ and $0.0001 \mathrm{~kg} / \mathrm{s}$ for $q_{\text {in }}$ and $725 \mathrm{~K}, 765 \mathrm{~K}$ and $810 \mathrm{~K}$ for $155 T$. Totally, the database contains an appreciable size of 979 experimental test points. 
The present database was subdivided in three subsets. A first subset (490 experimental

tests) is used to train the networks. A second one (245 experimental tests) is used to test the ANN models to determine when to stop the training stage. The third subset ( 244 experimental tests) is used to validate the performance of the selected model on unseen cases.

Each input or output parameter has been normalized relative to its minimum and maximum values observed in the data (according to Eq. (1)) to make the training procedure more efficient.

$$
X_{\text {norm }}=\frac{\left(X-X_{\min }\right)}{\left(X_{\max }-X_{\min }\right)}
$$

164 where $X$ is an arbitrary parameter, $X_{\text {norm }}$ is the normalized value, and $X_{\max }$ and $X_{\min }$ are the maximum and minimum values of $X$.

\subsection{Architecture and learning process of ANN models}

An artificial neural network model is composed of interconnected group of artificial neurons or nodes. The most frequently utilized network is the multilayer backpropagation neural network (BPNN) which is used in the present study. The BPNN structure consists of three layers, an input layer which receive data; an output layer which sends computed information; and one or more hidden layers to link input and output layer. All the neurons (nodes) in a layer are connected with all the neurons of the previous and the next layer. In general, the number of the nodes in the input and output layer are determined by the nature of the problem. The architecture of a typical 3-layer backpropagation neural network is shown in Fig. 3.

\section{Figure 3 should be placed here}


Mathematically, a 3-layer BPNN with $n, m$, and $p$ the number of input, hidden and output

neurons respectively, can be formulated as in the following:

$$
O_{k}=f\left(b_{k}+\sum_{j=1}^{m} f\left(b_{j}+\sum_{i=1}^{n} W_{i j} X_{i}\right) \times W_{j k}\right)
$$

181 where $X_{i}$ the input values of the network and $O_{k}$ are the output values; $b_{j}$, the hidden unit 182 biases; $b_{k}$, output nodes biases; $W_{i j}$, the connection weights between the input layer and the 183 hidden layer; $W_{j k}$, the connection weights between the hidden layer and the output layer; $f$ is a 184 transfer function. The sigmoid transfer function (Eq. (3)) was used in the present study.

$$
f(x)=\frac{1}{1+e^{-x}}
$$

186 Where $x$ is the excitation.

187 The learning process of BPNN is based on a series of connection weight adjustments in 188 order to minimize the gap (global error) between the outputs of the BPNN and the target 189 values [31]. Initially, all biases and connection weights are initialized to random values in the 190 range of $[-1,+1]$. Inputs are first propagated forward through each layer of the ANN. Errors 191 between outputs and target values are then propagated backwards and the connection weights 192 are modified according to a specific learning algorithm (delta rule) to reduce the overall error. 193 This process (forward-backward) is repeated until predicted outputs and target answers 194 coincide within a given tolerance [32].

195 The commonest convergence criterion is the average squared error (ASE) defined as:

$$
A S E=\frac{1}{p} \times \frac{1}{S} \times \sum_{q=1}^{s} \sum_{k=1}^{p}\left(t_{q k}-O_{q k}\right)^{2}
$$


where $t_{q k}$ and $O_{q k}$ are respectively the target and predicted value of the output node $\mathrm{k}$ for the pattern $q, p$ is the number of output nodes, and $s$ is the number of patterns. It should be noted that any level of agreement between predicted and target vectors can be achieved by providing a sufficient number of training cycles to be carried out. Such an overtraining is however detrimental to the capacity of the network to generalize from unseen data (a network that can accurately predict the output of the testing patterns is said to have generalized). It is thus preferable to calculate the ASE both on training and testing patterns during training cycles for optimum convergence: this process is called cross-validation (Fig. 4).

\section{Figure 4 should be placed here}

\section{Results and Discussion}

5.1 Optimum artificial neural network architecture

The determination of the ANN architecture constitutes one of the major tasks in the use of the ANN. The overall performance of an ANN is dependent on the numbers of hidden layers and hidden nodes. In the usual case of a 3-layer BPNN, the optimum number of hidden nodes can be determined by cross-validation in the same way as the optimum number of training cycles (Fig. 4).

In the present article, a neural network relating inputs $\left\{X_{1}, X_{2}, \ldots, X_{n}\right\}$ to outputs $\left\{O_{1}, O_{2}\right.$, $\left.\ldots, O_{p}\right\}$ and containing one hidden layer with $\mathrm{m}$ hidden nodes will be noted:

$$
\left\{O_{1}, O_{2}, \ldots, O_{p}\right\}=\mathrm{ANN}_{\mathrm{n} \square \mathrm{m} \square \mathrm{p}}\left\{X_{1}, X_{2}, \ldots, X_{n}\right\}
$$

217 In our case, the outlet operating pressure $\left(P_{\text {out }}\right)$ is sought as a function of $t, P_{i n}, q_{\text {in }}$ and $T$. So, 218 it is possible to compute $P_{\text {out }}$ by using a BPNN model with one node in the output layer (Eq. 219 (6)). It could be noticed that usually the pressure drop through the porous medium is investigated as a function of the through-flow rate. 
In this present study, the choice of the outlet pressure is preferred to limit the impact of experimental uncertainties due to the two pressure transducers (which are generally multiplied if compared to a single transducer). In addition, since the upstream pressure is an inlet parameter, looking at the pressure drop or at the pressure outlet is equivalent when focusing on the behaviour of the ANN model. As can be observed in Fig. 5, the optimal value of ASE was calculated while using 9 nodes in the hidden layer for our model.

$$
\left\{P_{\text {out }}\right\}=\mathrm{ANN}_{4-9-1}\left\{t, P_{\text {in }}, q_{\text {in }}, T\right\}
$$

The ASE values for the training, testing and validation phases for the optimal artificial neural network model $\left(\mathrm{ANN}_{4-9-1}\right)$ are respectively $0.000114,0.000101$ and 0.000132 .

\section{Figure 5 should be placed here}

\subsection{Discussion of the performance of the models}

The performance of the ANN model is evaluated by comparing target $\left(Y_{i}\right)$ and predicted $\left(\hat{Y}_{i}\right)$ values. Fig. 6 shows the comparison between the BPNN predicted values and the target values for $P_{\text {out }}$ on training, testing and validation data. Despite the pressure drop is the parameter of interest for engineering application, the present model focuses on the outlet pressure to clearly estimate the validity of the model. On the same graphs the best fit line through the origin is also plotted and the coefficient of determination $\mathrm{R}^{2}$ for this line is computed according to Eq. (7):

$$
R^{2}=1-\frac{\frac{1}{N} \sum_{i=1}^{N}\left(Y_{i}-\hat{Y}_{i}\right)^{2}}{\frac{1}{N-1} \sum_{i=1}^{N}\left(Y_{i}-\bar{Y}_{i}\right)^{2}}
$$

240 where $N$ is the number of data, $Y_{i}$ is the target value, $\hat{Y}_{i}$ is the value predicted by the model and $\overline{Y_{i}}$ is the mean of the $N$ target values. $R^{2}$ coefficients close to unity indicate a high degree 
of linearity between predicted and target values. Associated with a best fit line slope close to

unity, it indicates a high model prediction accuracy.

A basis of comparison for BPNN performance is usually sought in multiple linear regression [33], a more ubiquitous prediction tool in fluid flow through porous material research. Least square parameter fitting for a linear model expressing $P_{\text {out }}$ as a function of $t$, $P_{i n}, q_{i n}$, and $T$ (model 2) is performed on the same training database subset as for BPNN model. This model is tested to predict the never-seen data from the BPNN validation database subset. The lowest $R^{2}$ value is obtained for multiple linear regression model. It is also noted that the trend line deviates somewhat from the 1:1 line in the case of model 2 . The coefficients of determination $\left(R^{2}\right)$ for model 1 and model 2 are given in Table 1 . The model 2 seems to be less efficient than model 1 for predicting the variations of $P_{\text {out }}$. This result is expected: the physical phenomena captured in the database are complex and non-linear. In ANN non-linearity is accounted for by the use of transfer functions (Eq. (3)), while complexity can be controlled by varying the number of hidden nodes. In the present case, the artificial neural networks provide good and realistic predictions.

Figure 6 should be placed here

\section{Table 1 should be placed here}

An application of ANN is now proposed in the following part. Considering a constant inlet pressure at $3.6 \mathrm{MPa}$, it is now possible to investigate the chemical effect within the range of $725 \mathrm{~K}-810 \mathrm{~K}$. It should be noticed that fixing arbitrarily the inlet pressure imposes the outlet pressure to decrease, which simulate the pressure drop increase. The evolution of the predicted outlet pressure $\left(P_{\text {out }}\right)$ using BPNN as a function of time and temperature inside the 
porous medium is given in Fig. 7. It is found with model 1 that the predicted outlet pressure

decreases as a function of time. This expected result is due to the formation of carbon deposit (coke) on the surface and inside the porosities of the studied material. We can observe also that the model 1 predicted outlet pressure decreases when the temperature inside the porous medium increases. The density decreases due to the thermal rise and since the mass flow rate inside the medium is kept constant, the mean reactive fluid velocity increases. Thus, the outlet pressure decreases; which means that the pressure drop increases. This is clearly understandable when paying attention to the Brinkman equation. It is thus very important to note that the BPNN approach is able to reproduce physical variations. In particular, it is clear that the chemical effect strongly increases at $760 \mathrm{~K}$ and is clear at $770 \mathrm{~K}$.

\section{Figure 7 should be placed here}

The result of Fig. 7 can be not only related to thermal effect on density and velocity but also to the chemical effect. Indeed, the reactive fluid outlet pressure decreases as a function of time due to the formation of the coke on the surface and inside the studied material. The thermal effect may increase the fluid velocity within the porous material by enhancing coke formation and pore clogging (the lower the cross-section area, the higher the fluid velocity and the higher the pressure in case of constant mass flowrate configuration).

Measuring the pressure drop through porous material could be a way to get information of phenomena within the porous material where no direct microscopic measure seems to be possible for the fluid properties. In addition, these results should drive the engineering study of material cooling because ensuring constant cooling efficiency, thus constant fluid flow through the porous medium, clearly requires compensating higher pressure drop depending on the temperature seen by the solid materials. As a consequence, performance of pumping 
system should be designed to furnish this increasing need for upstream pressure if outlet

\section{Conclusion}

In this article, an artificial neural networks tool has been used to simulate the transient pressure drop of $\mathrm{n}$-dodecane under pyrolysis conditions and crossing a metallic porous material (Stainless steel). Based on experimental data, the optimum architecture of artificial neural network was trained and validated, in order to generalise the prediction of the pressure drop under clogging configurations not included in the database for difficult access reasons. The validation showed excellent performance of this ANN model for the prediction of dodecane transfer in the porous material $\left(\mathrm{R}^{2}>0.983\right)$. An example of application was presented to detect the temperature at which chemistry starts to strongly impact the fluid flow within the porous medium. It was found that a turning point around $760 \mathrm{~K}-770 \mathrm{~K}$ has to be expected in terms of clogging when using n-dodecane at 3.6 MPa in stainless steel medium.

This study is a contribution to the growing evidence of the benefits of ANN models in Aeronautical engineering. This important result may be applied to automate pressure drop estimations, which are used in space flight applications, without prior knowledge of material parameters and particularly for materials with transient changing properties.

\section{REFERENCES}

[1] M. Kuhn, H. Hald, Application of transpiration cooling for hot structures, RESPACE: Key Technologies for Reusable Space Systems, Note N. Fl. Mech. Mul. D., 2008, 98, $82-103$.

[2] N. Gascoin, High temperature and pressure reactive flows through porous media, Int. J. Multiphas. Flow, 2011, 37, 24-35. 
[3] T. Langener, J.V Wolfersdorf, J. Steelant, Experimental investigations on transpiration

6 cooling for scramjet applications using different coolants, AIAA J., 2011, 49(7), 14091419.

[4] N. Gascoin, G. Fau, P. Gillard, M. Kuhn, M. Bouchez, J. Steelant, Comparaison of two permeation test benches and two determination methods for Darcy's and Forchheimer's permeabilities, J. Porous Media, 2012, 15, 705-720.

[5] B. Zhang, L.M. Lei, X.L. Jiang, Z.M. Song, X. Huang, G.P. Zhang, On Temperature and Strain Rate Dependent Strain Localization Behavior in Ti-6.5Al-3.5Mo-1.5Zr0.3Si Alloy, J. Mater. Sci. Technol., 2013, 29(3), 273-278.

[6] L. Romagnosi, N. Gascoin, E. El-Tabach, I. Fedioun, M. Bouchez, J. Steelant, Pyrolysis in Porous Media: Part 1. Numerical model and parametric study, Energ. Convers. Manage., 2013, 68, 63-73.

[7] E. El Tabach, N. Gascoin, P. Gillard, Neural-Network Metamodelling for the Prediction of the Pressure Drop of a Fluid Passing Through Metallic Porous Medium, J. of Porous Media, 2014, 17, 431-438.

[8] Y. Zhou, H. Xiang, Z. Feng, Theoretical Investigation on Mechanical and Thermal Properties of a Promising Thermal Barrier Material: $\mathrm{Yb}_{3} \mathrm{Al}_{5} \mathrm{O}_{12}$, J. Mater. Sci. Technol., 2014, 30(7), 631-638.

[9] S.D. Ji, Y.Y. Jin, Y.M. Yue, S.S. Gao, Y.X. Huang, L. Wang, Effect of Temperature on Material Transfer Behavior at Different Stages of Friction Stir Welded 7075-T6 Aluminum Alloy, J. Mater. Sci. Technol., 2013, 29(10), 955-960.

[10] G. Fau, N. Gascoin, P. Gillard, M. Bouchez, J. Steelant, Fuel pyrolysis through porous media: Coke formation and coupled effect on permeability, J. Anal. Appl. Pyrol., 2012, $95,180-188$. 
[11] O. Herbinet, P.M. Marquaire, F. Battin-Leclerc, R. Fournet, Thermal decomposition of n-dodecane: Experiments and kinetic modeling, J. Anal. Appl. Pyrol., 2007, 78, 419429.

[12] F. Billaud, F. Baronnet, C.P. Gueret, Thermal coupling of methane in a tabular flow reactor: parametric study, Ind. Eng. Chem. Res., 1993, 32, 1549-1554.

[13] D.B. Murphy, R.W. Caroll, J.E. Klonowski, Analysis of products of high-temperature pyrolysis of various hydrocarbons, Carbon, 1997, 35, 1819-1823.

[14] G. Liu, Y. Han, L. Wang, X. Zhang, Z. Mi, Supercritical thermal cracking of Ndodecane in presence of several initiative additives: products distribution and kinetics, Energy Fuels, 2008, 22, 3960-3969.

[15] G. Fau, N. Gascoin, J. Steelant, Hydrocarbon pyrolysis with a methane focus: A review on the catalytic effect and the coke production, J. Anal. Appl. Pyrol., 2014, 108, 1-11.

[16] P. Zhou, B.L. Crynes, Thermolytic reactions of dodecane, Ind. Eng. Chem. Proc.DD., $1986,25,508-514$.

[17] D.S. Aribike, A.A. Susu, Thermal cracking of n-Butane and a light hydrocarbon mixture, J. Anal. Appl. Pyrol., 1988, 14, 37-48.

[18] S. Wauters, G.B. Marin, Computer generation of a network of elementary steps for coke formation during the thermal cracking of hydrocarbons, Chemical Engineering Journal, $2001,82,267-279$.

[19] J.P. Chakraborty, D. Kunzru, High pressure pyrolysis of n-heptane, J. Anal. Appl. Pyrol., 2009, 86, 44-52.

[20] S.M. Sadrameli, Thermal/catalytic cracking of hydrocarbons for the production of olefins: A state-of-the-art review 1: Thermal cracking review, Fuel, 2015, 140, $102-$ 115. 
[21] S. Marinkovic, Carbon/carbon composites prepared by chemical vapor infiltration-15 years later, Carbon, 1991, 29, 747-752.

[22] H.J. Li, X.H. Hou, Y.X. Chen, Densification of unidirectional carbon-carbon composites by isothermal chemical vapor infiltration, Carbon, 2000, 38, 423-427.

[23] X. Wu, R. Luo, J. Zhang, Q. Li, Y. Ni, Kinetics of thermal gradient chemical vapor infiltration of large-size carbon/carbon composites with vaporized kerosene, Mater. Chem. Phys., 2009, 113, 616-621.

[24] H. Deng, K. Li, H. Li, X. Li, L. Zhang, W. Cao, Densification behavior and microstructure of carbon/carbon composites prepared by chemical vapor infiltration from xylene at temperatures between 900 and $1250{ }^{\circ} \mathrm{C}$, Carbon, 2011, 49, 2561-2570.

[25] J. Ren, K. Li, S. Zhang, X. Yao, S. Tian, Preparation of carbon/carbon composite by pyrolysis of ethanol and methane, Mater. Design, 2015, 65, 174-178.

[26] N. Gascoin, P. Gillard, S. Bernard, M. Bouchez, Charaterisation of coking activity during supercritical hydrocarbon pyrolysis. Fuel Process. Technol., 2008, 89, 14161428

[27] E. El Tabach, L. Lancelot, I. Shahrour, Y. Najjar, Use of artificial neural network simulation metamodelling to assess groundwater contamination in a road project, Math. Comput. Model., 2007, 45, 766-776.

[28] V. Arumugam†, R. Naren Shankar, B.T.N. Sridhar, A. Joseph Stanley, Ultimate Strength Prediction of Carbon/Epoxy Tensile Specimens from Acoustic Emission Data, J. Mater. Sci. Technol., 2010, 26(8), 725-729.

[29] A. Nazari, S. Riahi, Computer-aided Prediction of the $\mathrm{ZrO}_{2}$ Nanoparticles Effects on Tensile Strength and Percentage of Water Absorption of Concrete Specimens, J. Mater. Sci. Technol., 2012, 28(1), 83-96. 
385 [30] J.P.C. Kleijnen, Kriging metamodeling in simulation: A review. Eur. J. Oper. Res., 1 $2386 \quad 2009,192,707-716$.

[31] Y.M. Najjar, I.A. Basheer, M.N. Hajmeer, Computational neural networks for predictive microbiology: i. Methodology, Int. J. Food Microbiol., 1997, 34, 27-49.

[32] R.O. Duda, P.E. Hart, D.G. Stork, Pattern Classification, Wiley, NY, USA, 2001, ISBN: 978-0-471-05669-0

[33] B.G. Tabachnick, L.S. Fidell, Using multivariate statistics (6 ${ }^{\text {th }}$ ed.), Boston: Pearson Education, 2013, ISBN-13: 9780205849574. 
394 Table $1 R^{2}$ values between target and predicted outputs for all models.

Fig. 1 Schematic of the permeation test cell with porous material and associated measures.

Fig. 2 Measured pressure drop variations with the measured fuel mass flow rate and experiment time for $\mathrm{T}=725 \mathrm{~K}$.

Fig. 3 Architecture of a typical multilayer BPNN.

Fig. 4 Convergence criterion and optimum network architecture.

Fig. 5 Average squared error (ASE) variations with the number of hidden nodes for the testing data subset.

Fig. 6 Comparison between target and predicted values for $\mathrm{P}_{\text {out }}$ using BPNN (model 1) and multi-linear regression (model 2) for all data subset.

Fig. 7 Model 1 predicted transient $P_{\text {out }}$ variations with the temperature for $q_{\text {in }}=0.04 \mathrm{~g} / \mathrm{s}$ and $\mathrm{P}_{\mathrm{in}}=3.6 \mathrm{MPa}$. 
Table $1 R^{2}$ values between target and predicted outputs for all models.

\begin{tabular}{lll}
\hline$R^{2}$ & Neural network (model 1) & Multiple linear regression (model 2) \\
\hline Training phase & 0.986 & 0.897 \\
Testing phase & 0.986 & 0.878 \\
Validation phase & 0.983 & 0.891 \\
\hline
\end{tabular}




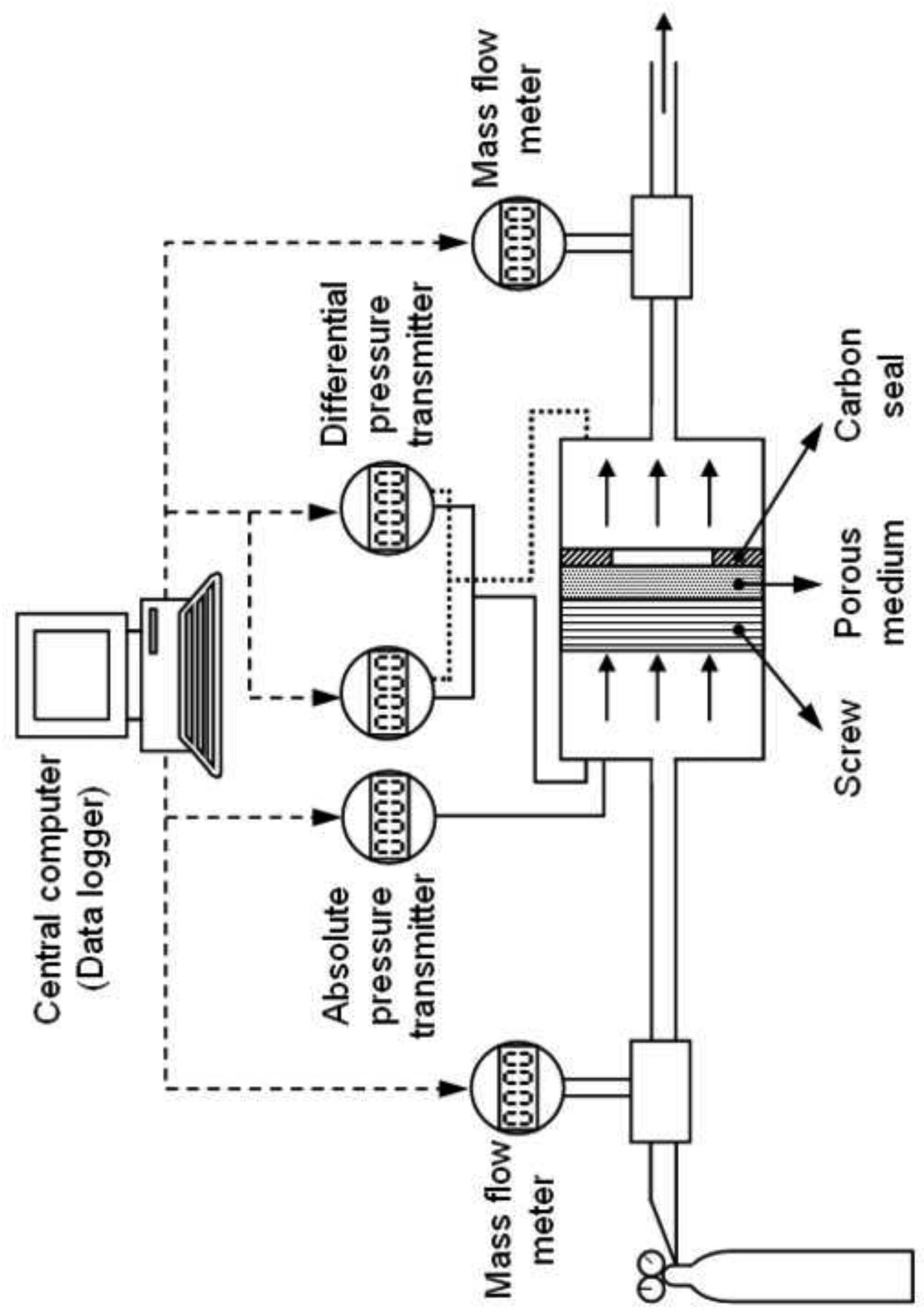




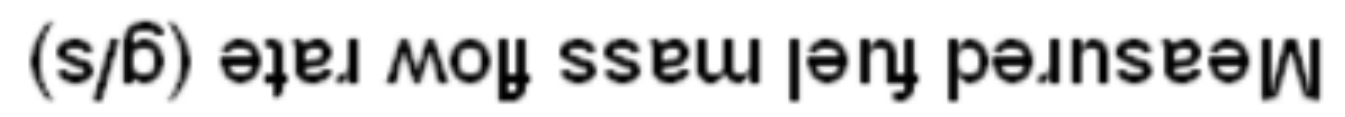

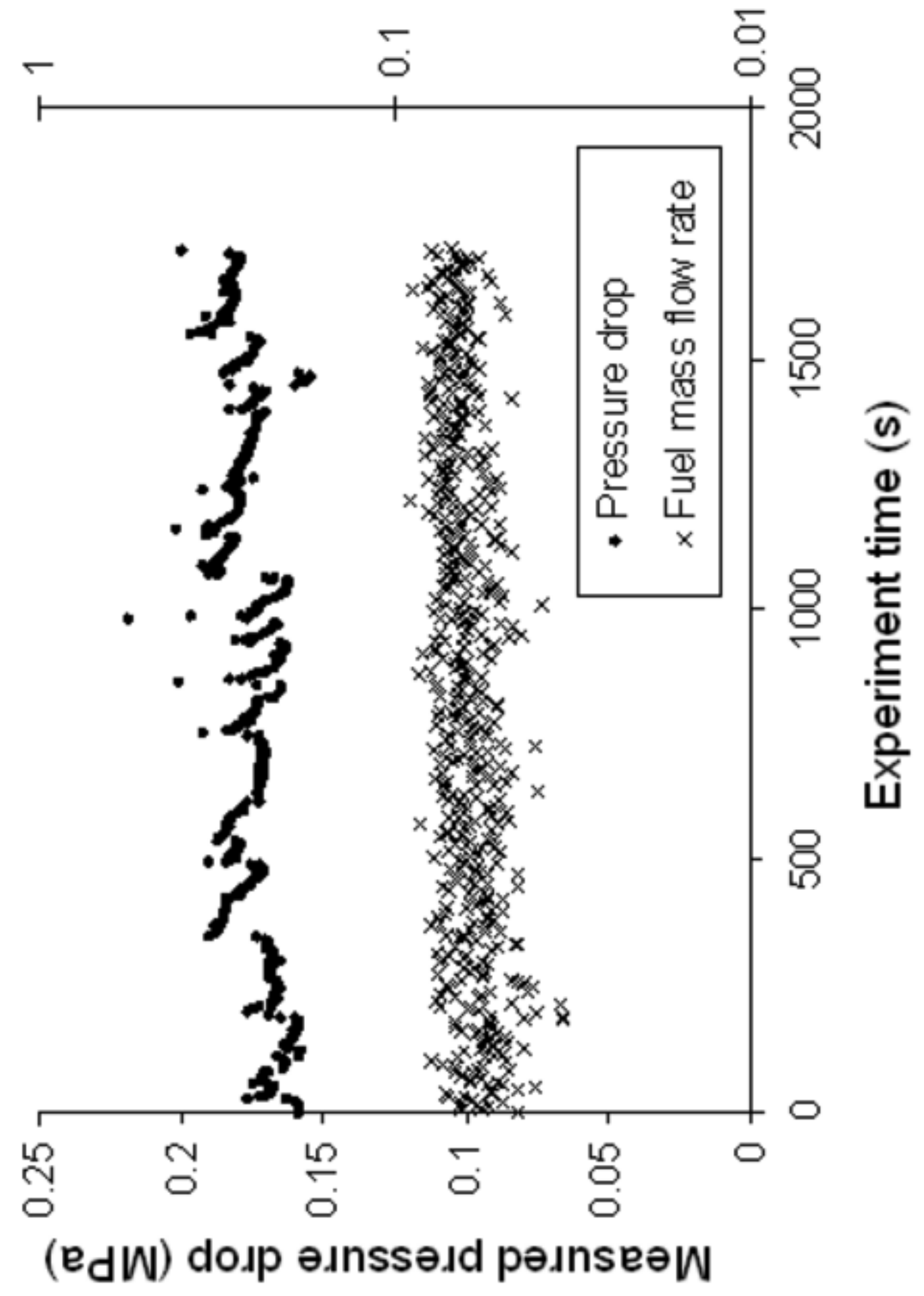


Input layer.

( $\mathrm{n}$ nodes) Hidden layer.

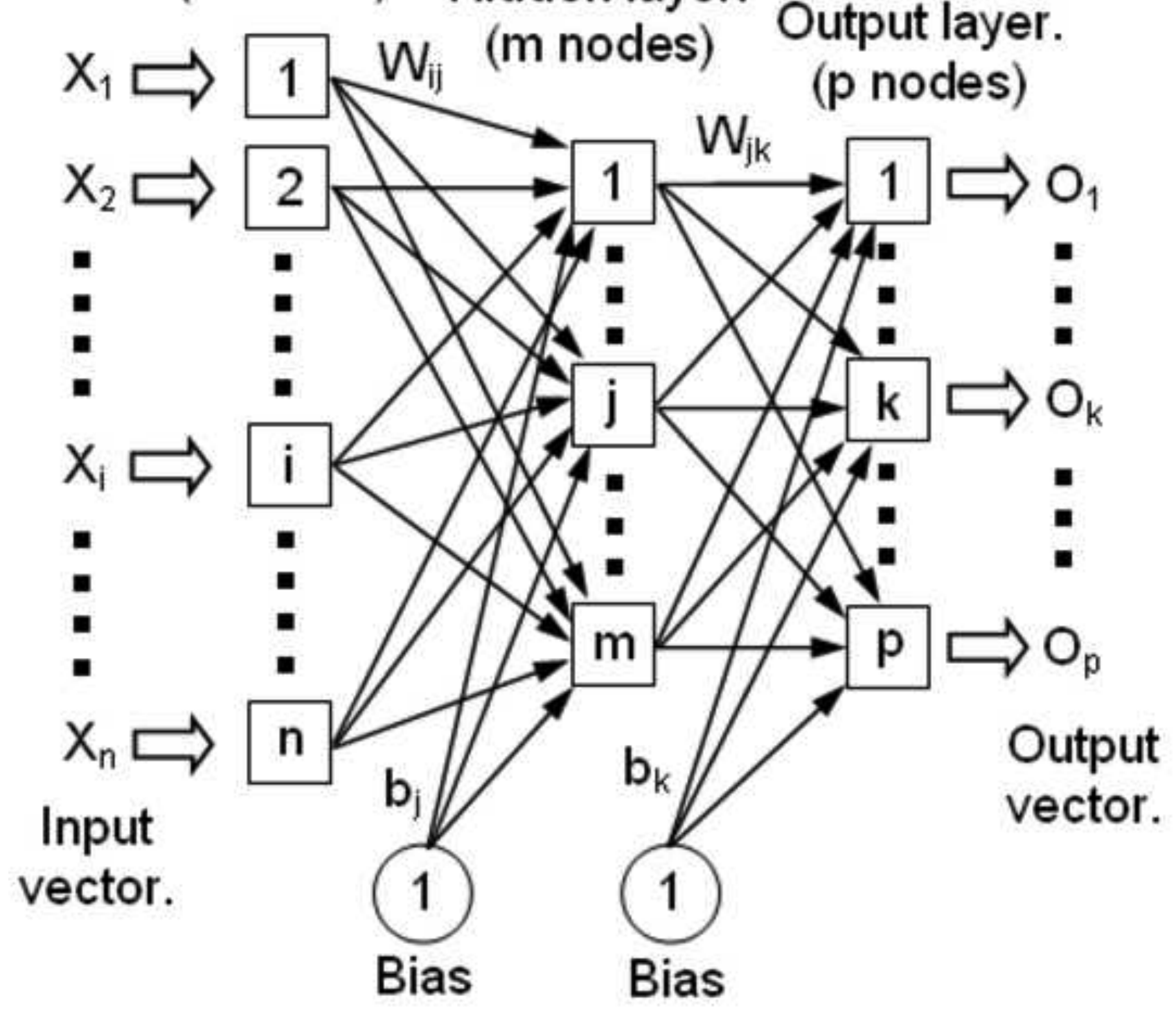




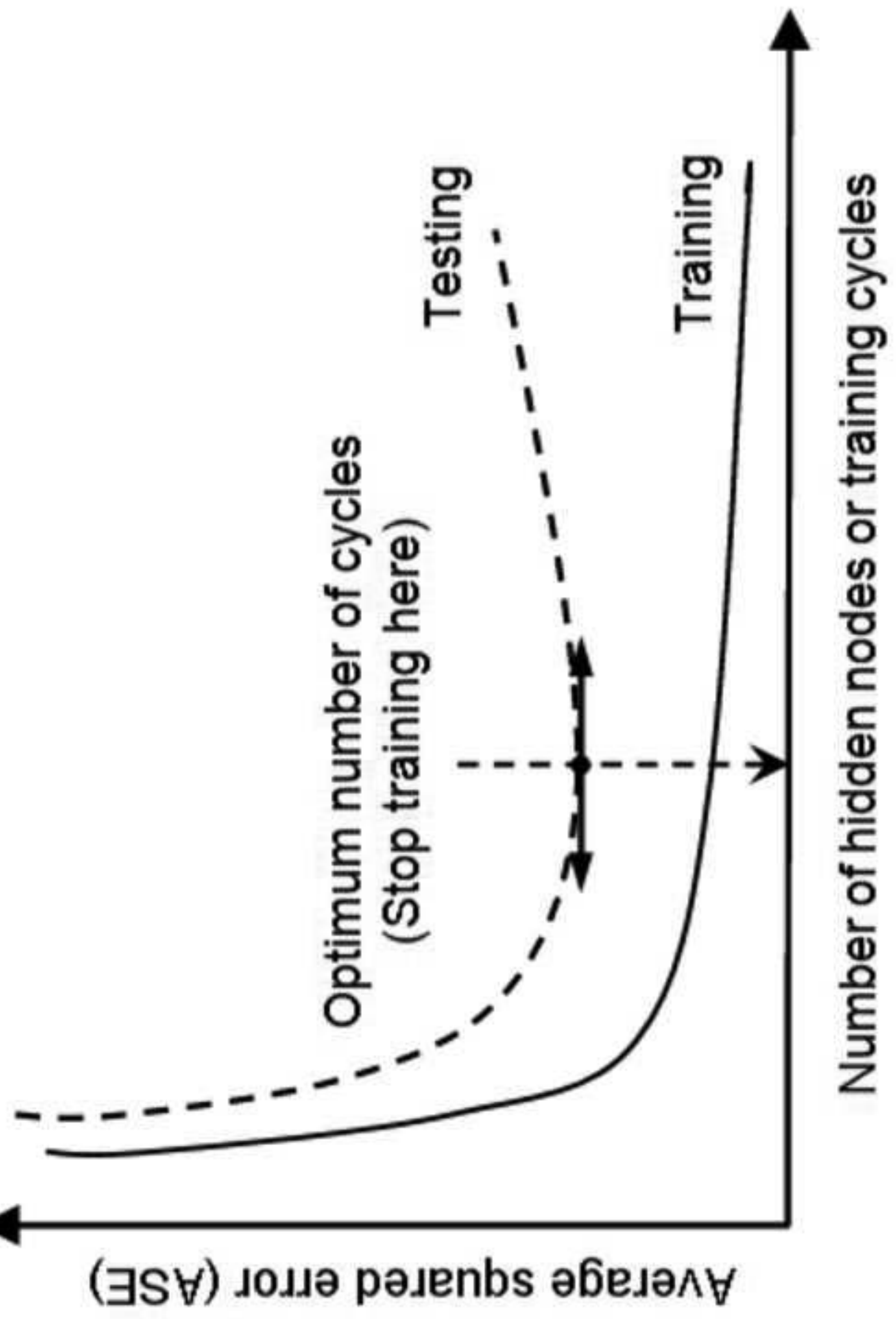

$\stackrel{9}{\circ}$

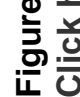




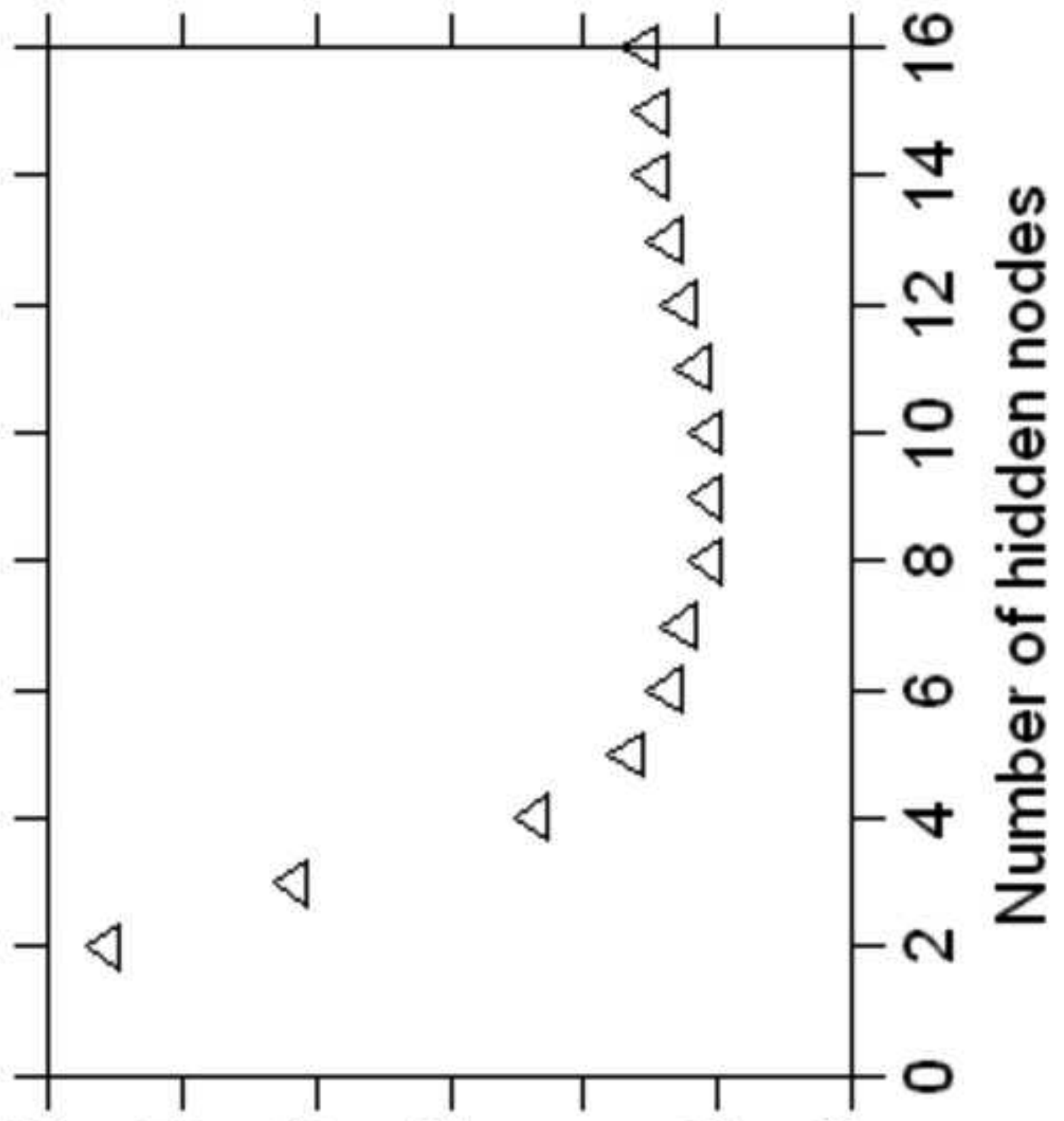

ᄂ

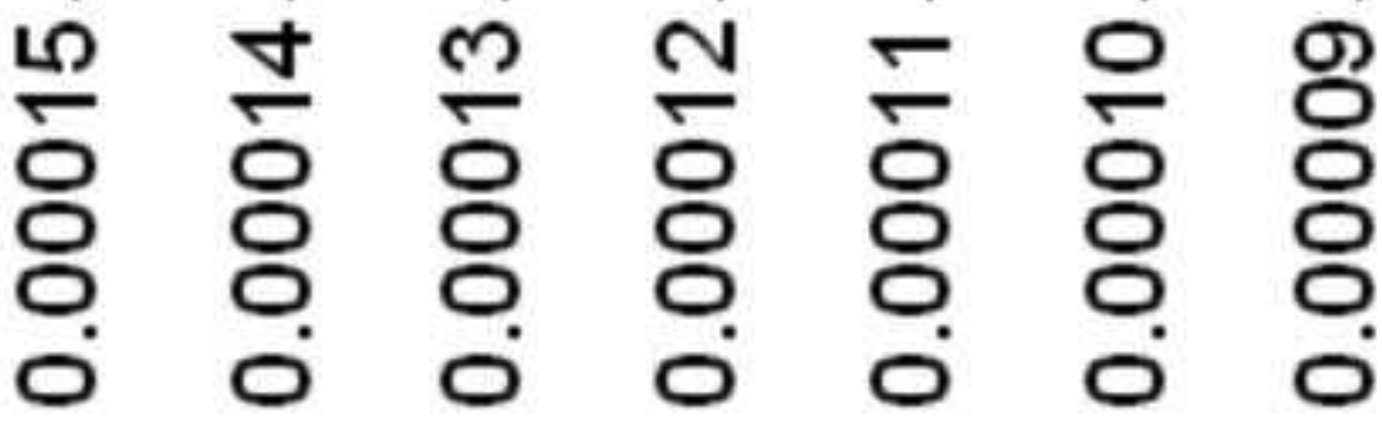

$\exists S \forall$ 

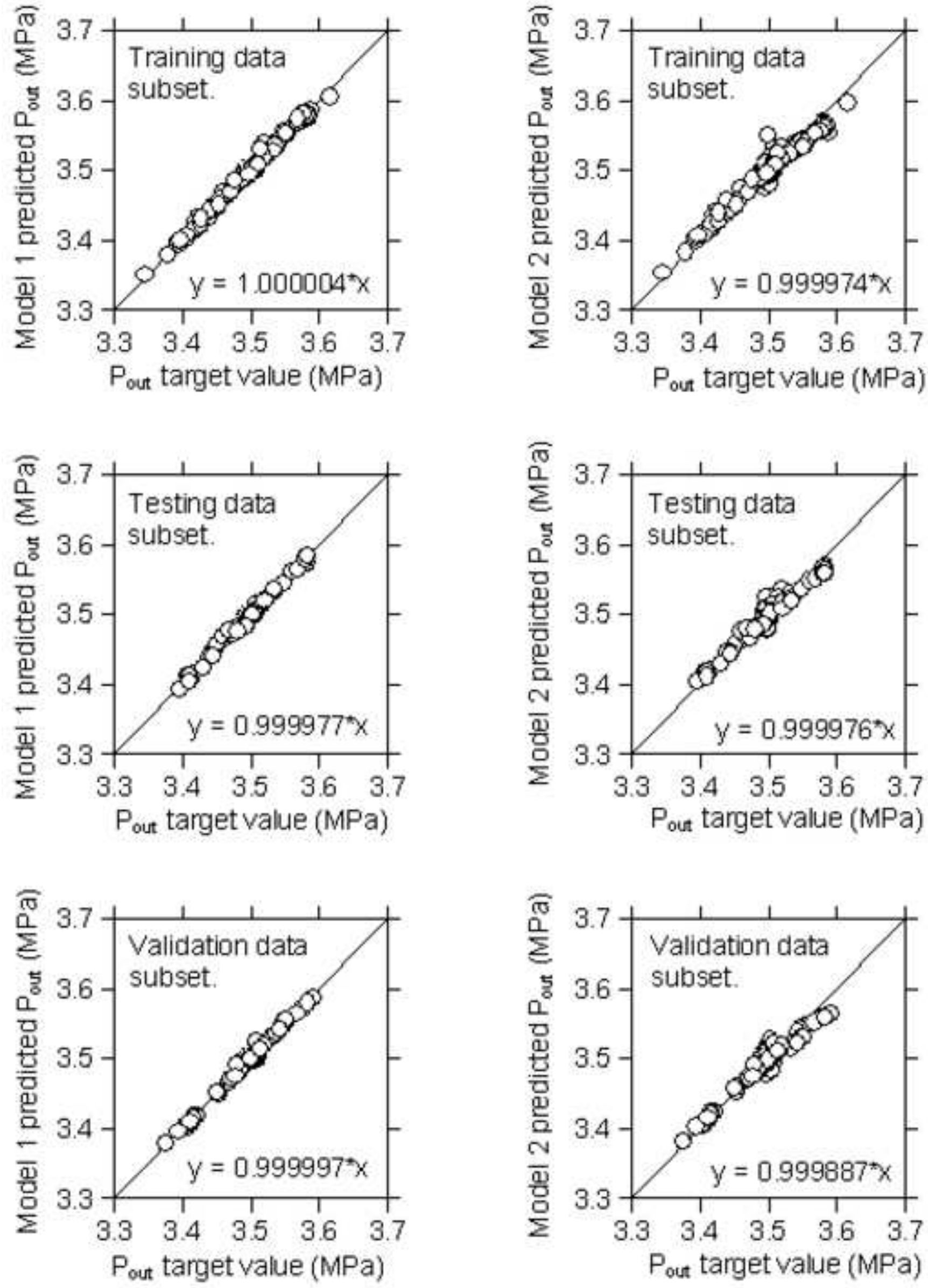


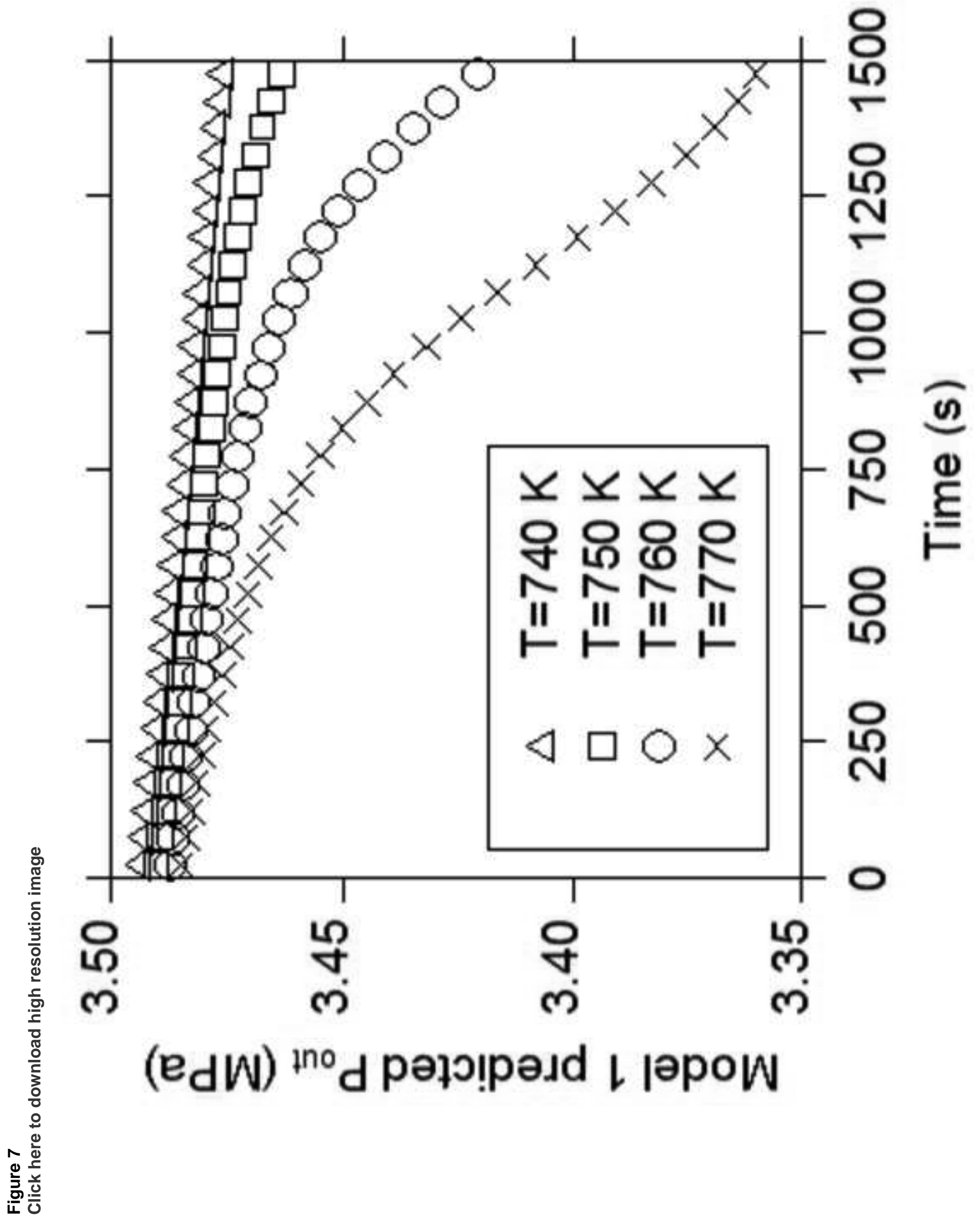




\section{Highlights :}

- We modeled the transient pressure drop of n-dodecane under pyrolysis conditions.

- We found a good agreement between the numerical results and the experimental data.

- We found that the developed model is able to reproduce physical variations.

- The model has been applied successfully on a series of examples. 